\title{
ATENDIMENTO À GESTANTE TRAUMATIZADA
}

\author{
TRAUMA IN PREGNANCY
}

Gerson Alves Pereira Júnior'; Luiz Fernando Haikel Júnior²; João Marcos Camillo Atique²; Ernani Jodi Nakamura²; Aníbal Basile-Filho ${ }^{3} \&$ José Ivan de Andrade ${ }^{4}$

\begin{abstract}
${ }^{1}$ Médico Assistente da Unidade de Emergência do Hospital das Clínicas; ${ }^{2}$ Graduandos, Membros da Liga do Trauma do Centro Acadêmico Rocha Lima; ${ }^{3}$ Professor Associado do Departamento de Cirurgia, Ortopedia e Traumatologia e Chefe da Disciplina de Terapia Intensiva; ${ }^{4}$ Professor Doutor do Departamento de Cirurgia, Ortopedia e Traumatologia. Faculdade de Medicina de Ribeirão Preto (FMRP) da Universidade de São Paulo (USP).

CorrespondênCIA: Dr. Gerson Alves Pereira Júnior - Rua Iguape, 747, ap. 12 C - Jardim Paulista - CEP: 14090-000 - Ribeirão Preto - SP. email: gersonapj@netsite.com.br
\end{abstract}

PEREIRA Jr. GA; HAIKEL Jr. LF; ATIQUE JMC; NAKAMURA EJ; BASILE-FILHO A \& ANDRADE JI. Atendimento à gestante traumatizada. Medicina, Ribeirão Preto, 32: 282-289, jul./set. 1999.

RESUMO: Este artigo faz uma revisão das alterações anatômicas e fisiológicas que ocorrem durante a gestação e estabelece os princípios da abordagem à gestante traumatizada, delineando os aspectos da monitorização materna e fetal e as patologias próprias da gestação. Diferenciam-se os traumas contusos e penetrantes, discutindo-se o papel do lavado peritoneal diagnóstico e da laparotomia exploradora. Discutem-se a abordagem da gestante em parada cardiorrespiratória e as indicações de cesárea perimorte, bem como os indícios de violência doméstica.

UNITERMOS: Lesões. Gravidez. Cesárea. Morte Fetal. Traumatismos Abdominais. Violência Doméstica.

\section{INTRODUÇÃO}

A possibilidade de gravidez deve ser considerada em qualquer paciente do sexo feminino na faixa etária entre os 10 e 50 anos, devendo-se sempre excluir tal possibilidade, de modo objetivo, nestes pacientes ${ }^{(1)}$.

A incidência de trauma durante a gravidez situa-se em torno de $6 \%{ }^{(2,3)}$.

A gestante traumatizada é uma paciente singular, porque são vitimadas duas pessoas simultaneamente. Ademais, as adaptações fisiológicas do organismo materno durante a gravidez alteram o padrão normal de resposta frente às diferentes variáveis envolvidas no trauma ${ }^{(1 / 4)}$. Estas alterações da estrutura e função orgânicas podem influenciar a avaliação da gestante traumatizada pela alteração dos sinais e sintomas das lesões, alterar a abordagem e a resposta à ressuscitação volêmica, bem como o resultado dos testes diagnósticos. A gestação pode afetar, também, o padrão e a gravidade das lesões. ${ }^{(1)}$

As prioridades no atendimento e tratamento da gestante traumatizada são as mesmas da paciente não grávida ${ }^{(1,4)}$. O melhor cuidado para com o feto é prover um tratamento adequado para a mãe, uma vez que a vida do feto é totalmente dependente da integridade anatomofisiológica materna ${ }^{(1,4)}$.

\section{ALTERAÇÕES ANATÔMICAS E FISIO- LÓGICAS DO ORGANISMO MATERNO DURANTE A GESTAÇÃO}

O útero persiste como um órgão intrapélvico até a $12^{\mathrm{a}}$ semana de gestação. Está na altura do umbigo entre 20 e 22 semanas e do gradeado costal após 
36 semanas. Com o crescimento uterino, o intestino delgado fica restrito ao abdome superior. Ao crescer e ultrapassar os limites da pelve, o útero sai de um ambiente anatomicamente "protegido", tornando-se mais vulnerável ao trauma ${ }^{(1,4)}$.

O líquido amniótico protege o feto dos traumas externos; porém, para a mãe, pode se constituir em fonte de embolia e coagulação intravascular dissemi$\operatorname{nada}^{(1,4)}$.

A falta de um tecido conjuntivo elástico na placenta predispõe o seu descolamento no trauma direto sobre o abdome ${ }^{(1,4)}$.

As alterações hematológicas ocorrem tanto no volume sangüíneo, quanto na sua composição.

O volume plasmático sofre um incremento progressivo, chegando a 45 a $50 \%$ por volta da $32^{\mathrm{a}}$ semana de gestação ${ }^{(1,4)}$. A quantidade de hemácias aumenta e, ao final da gestação, chega ao nível de 33\% maior do que na mulher não grávida ${ }^{(3)}$. $\mathrm{O}$ maior aumento relativo do volume plasmático em relação à massa de eritrócitos leva à chamada "anemia fisiológica da gravidez", cujo pico ocorre em torno da $28^{a}$ semana de gestação ${ }^{(1,2,3)}$. No final da gestação, o hematócrito está em torno de 31 a $35 \%^{(1 / 4)}$.

O aumento global, no volume sangüíneo, chega a $48 \%$ e é proporcional ao número de fetos, sendo de aproximadamente $1600 \mathrm{ml}$ na gravidez única e de $2000 \mathrm{ml}$ na gravidez gemelar ${ }^{(3)}$. Na vigência de hemorragia, uma perda de 30 a $35 \%$ do volume sangüíneo pode ser pouco sintomática na gestante. A hipotensão arterial é, usualmente, um sinal tardio ${ }^{(1,4)}$.

O número de leucócitos está aumentado no sangue periférico, sendo comum encontrarmos valores em torno de $20.000 / \mathrm{mm}^{3}$, sem alteração na contagem diferencial de glóbulos brancos ${ }^{(1,3,4)}$.

As concentrações de todos os fatores da coagulação estão aumentadas na gestação, exceto dos fatores XI e XIII e antitrombina III. O aumento do fibrinogênio plasmático e dos fatores VII, VIII e IX, associado à diminuição do fator ativador do plasminogênio, atua como predisponente da coagulação (diminui o tempo de protrombina e o tempo de tromboplastina parcial ativada, mas não altera o tempo de coagulação nem o tempo de sangramento $)^{(1,3,4)}$. Os níveis de fibrinogênio atingem $600 \mathrm{mg} / \mathrm{dL}$ próximo ao termo da gestação, sendo considerados anormais níveis menores que $400 \mathrm{mg} / \mathrm{dL}$. Estas alterações da composição sangüínea resultam em um estado de hipercoagulabilidade que, em associação com estase venosa e trauma das paredes venosas, produzem um risco aumentado para tromboembolismo.
A albumina sérica está diminuída (2,2 a 2,8 $\mathrm{g} / \mathrm{dL}$ ), levando a uma queda na proteína sérica total em, aproximadamente, $1 \mathrm{~g} / \mathrm{dL}^{(1)}$. A osmolaridade sérica permanece em torno de $280 \mathrm{mOsm} / \mathrm{L}$ durante toda a gestação ${ }^{(1)}$.

O débito cardíaco materno aumenta, a partir da $10^{\mathrm{a}}$ semana de gestação, chegando ao auge no final do $2^{\circ}$ trimestre ${ }^{(1,3,4)}$. Tal aumento, de aproximadamente 4,5 para 6 litros/minuto (30 a 45\%), decorre do aumento do volume plasmático e da diminuição da resistência vascular do útero e da placenta, que durante o $3^{\circ}$ trimestre chega a receber $20 \%$ do débito cardíaco. Este débito cardíaco elevado é mantido até retornar aos níveis não gravídicos em torno de duas a quatro semanas pós-parto ${ }^{(3)}$. As pressões de enchimento cardíaco (pressão venosa central e pressão na artéria pulmonar) não se alteram durante a gestação. Na posição supina, pode ocorrer uma queda de 30 a $35 \%$ do débito cardíaco, pela diminuição do retorno venoso dos membros inferiores causada pela compressão do útero sobre a veia cava inferior ${ }^{(1,4)}$.

As alterações na resistência vascular, durante a gestação, ocorrem por fatores mecânicos e hormonais ${ }^{(3)}$. O desenvolvimento da circulação uteroplacentária cria um importante circuito de baixa resistência que reduz a pós-carga cardíaca ${ }^{(3)}$. Os elevados níveis de estrógeno e progesterona, bem como das prostaglandinas, causam a vasodilatação periférica da gravidez ${ }^{(3)}$.

A frequiência cardíaca materna aumenta a partir da $7^{\text {a }}$ semana de gestação. Ao final do primeiro trimestre, o aumento é da ordem de 15 a 20 batimentos por minuto ${ }^{(1,4)}$. Esta alteração deve ser considerada na interpretação da taquicardia em resposta à hipovolemia ${ }^{(1)}$.

As pressões sistólica e diastólica diminuem durante o $2^{\mathrm{o}}$ trimestre, quando há uma queda de $05 \mathrm{a}$ $15 \mathrm{mmHg}$ nos valores basais, sendo que, próximo ao termo, a pressão arterial retorna, gradualmente, aos níveis normais ${ }^{(3,4)}$.

Os valores da pressão arterial são maiores em decúbito lateral do que na posição supina. Nesta, o útero obstrui a veia cava inferior (após 20 semanas de gestação) e diminui o retorno venoso ${ }^{(2,4)}$.

O eixo cardíaco está desviado para a esquerda em torno de $15^{\mathrm{o}(1,4,5)}$. A onda $\mathrm{T}$ está invertida em DIII, aVF e nas derivações precordiais ${ }^{(1)}$. A ocorrência de focos ectópicos é comum na gestação ${ }^{(1,4)}$.

O volume minuto respiratório eleva-se em $50 \%$, como resultado do aumento do volume corrente. Isto se deve aos elevados níveis de progesterona, que é um estimulante do centro respiratório ${ }^{(1,4)}$. 
As alterações anatômicas da cavidade torácica com elevação das cúpulas diafragmáticas diminui a capacidade residual funcional (soma dos volumes residual e de reserva expiratória) em cerca de $25 \%{ }^{(3)}$. A redução na capacidade residual funcional predispõe a paciente a ter atelectasias, se houver qualquer patologia.

A hipocapnia ( $\mathrm{p}_{\mathrm{a}} \mathrm{CO}_{2}$ em torno de $30 \mathrm{mmHg}$ ) é comum no final da gestaçãoo ${ }^{(2,3)}$. Os níveis de bicarbonato sérico estão diminuídos cerca de $4 \mathrm{mEq} / \mathrm{ml} \mathrm{e}$ o pH arterial está elevado, resultando em um estado crônico de alcalose respiratória compensada ${ }^{(2,5)}$. A diminuição da capacidade tampão do sangue pode agravar a acidose do choque circulatório ${ }^{(1)}$.

A soma destas alterações aumenta a profundidade da respiração, mas não a sua freqüência, o que ajuda a suprir o aumento do consumo de oxigênio, que se eleva de 20 para $40 \mathrm{ml} / \mathrm{min}$ na segunda metade da gestação ${ }^{(3)}$.

Durante a gestação, o metabolismo e o consumo de oxigênio aumentam em torno de $20 \%$. O aumento do consumo de oxigênio deve-se às maiores necessidades metabólicas das mamas, do útero, da placenta e do crescimento fetal, associado ao maior trabalho respiratório e muscular, causado pela modificação do peso materno ${ }^{(6)}$. Tal aumento, no consumo de oxigênio, associado à diminuição na capacidade residual funcional, diminui a reserva de oxigênio da mãe e, subseqüentemente, aumenta o risco de hipóxia para o feto em resposta à hipoventilação ou apnéia materna.

A $\mathrm{p}_{\mathrm{a}} \mathrm{O}_{2}$ normal na gestante varia de 101 a 108 mmHg no início da gravidez e cai para 90 a 100 mmHg próximo ao termo ${ }^{(3)}$.

Em uma pessoa saudável, todas as regiões do pulmão estão abertas no final de uma inspiração profunda. Normalmente, a capacidade residual funcional excede o volume crítico de oclusão. Durante a gestação, o volume de oclusão não se altera, mas a capacidade residual funcional declina consideravelmente, permanecendo segmentos pulmonares colabados ao final da inspiração, o que reduz a complacência pulmonar e aumenta o "shunt" intrapulmonar. Isto explica porque os processos pneumônicos têm maior morbidade e mortalidade na gestante ${ }^{(3)}$.

As reduções da pressão do esfíncter inferior do esôfago e da motilidade gástrica favorecem o vômito. Por isto, o estômago deve ser descomprimido precocemente, assumindo que a gestante esteja com o estômago cheio, diminuindo o risco de aspiração do conteúdo gástrico ${ }^{(2,4,5)}$.
O intestino delgado é deslocado para o abdome superior pelo crescimento uterino, enquanto o fígado e o baço permanecem em posição normal ${ }^{(4)}$.

A placenta produz fosfatase alcalina, elevando os níveis séricos desta enzima ${ }^{(5)}$.

A taxa de filtração glomerular e o fluxo sangüíneo renal estão aumentados na gestação. Há queda dos níveis plasmáticos de creatinina e uréia, à metade dos valores normais ${ }^{(4,5)}$. Glicosúria é comum durante toda a gestação.

Os cálices, a pelve renal e os ureteres estão dilatados, podendo persistir tal dilatação por várias semanas após o término da gravidez ${ }^{(5)}$. Devido à freqüente dextrorotação uterina, a dilatação, geralmente, é mais pronunciada à direita.

$\mathrm{O}$ aumento do útero resulta em compressão vesical. O tônus da bexiga diminui, aumentando sua capacidade e retardando seu esvaziamento. A estase urinária resultante facilita a infecção ${ }^{(2,5)}$.

A hipófise tem seu peso aumentado em 30 a $50 \%$ (1). Se ocorrer choque circulatório, pode haver necrose da porção anterior da glândula, levando ao hipopituitarismo (Síndrome de Sheeran) $)^{(2,5)}$.

A sínfise púbica se alarga a partir $07^{\circ}$ mês $(04$ a 08 milímetros), assim como as articulações sacroilíacas, devendo ser consideradas tais alterações na interpretação da radiografia simples de pelve $\mathrm{e}^{(1)}$.

A eclâmpsia pode simular trauma cranioencefálico com convulsões. A presença de hipertensão, hiperreflexia, proteinúria e edema periférico associam-se a eclâmpsia. A ocorrência de proteinúria é decisiva no diagnóstico diferencial ${ }^{(4)}$.

\section{MECANISMOS DO TRAUMA}

\subsection{Trauma abdominal contuso}

A causa mais frequiente é o acidente por veículo motorizado, seguida por quedas e agressão direta sobre o abdome ${ }^{(3,6)}$.

O uso do cinto de segurança diminui a incidência de lesões maternas e a morte por prevenir a ejeção do veículo. Assim, as informações sobre a utilização e o tipo de cinto de segurança usado são importantes na avaliação global, pois os cintos de fixação abdominal ensejam maior risco de compressão uterina com possível ruptura ou descolamento de placenta ${ }^{(1,7)}$. Os cintos de três pontos dissipam as forças numa superfície maior, o que os torna mais eficientes, porém, a sua porção abdominal deve ficar abaixo das cristas ilíacas ântero-superiores e não sobre o abdome ${ }^{(7,8)}$. 
Hemorragias retroperitoneais graves secundárias ao trauma da pelve e do abdome inferior ocorrem mais frequientemente na grávida do que na paciente não gestante, devido ao maior tamanho dos órgãos pélvicos e ao maior fluxo sangüíneo para esta área ${ }^{(3,8)}$.

O trauma do intestino delgado ocorre menos freqüentemente durante a gestação, pois as alças intestinais são protegidas pelo útero aumentado de ta$\operatorname{manho}^{(1,2,6)}$.

A ruptura uterina traumática é pouco frequiente. O quadro clínico varia de sinais e sintomas mínimos, peritonismo, até hemorragia maciça e choque circulatório $^{(2,8)}$. A posição fetal anômala, extremidades estendidas ou ar livre intraperitoneal são evidências radiológicas de ruptura uterina. A suspeita de ruptura do útero demanda exploração cirúrgica imedia$\operatorname{ta}^{(1,3,7,8,9)}$.

O descolamento da placenta após trauma contuso é uma das causas de morte fetal e pode ocorrer mesmo após pequenos traumas na gestação avançada. Os achados no exame físico incluem dor abdominal, hipertonia uterina, sangramento vaginal, altura uterina em expansão, alterações da frequiência cardíaca fetal e choque materno. Com o descolamento de $25 \%$ da superfície placentária, o sangramento vaginal e o trabalho de parto prematuro podem ocorrer ${ }^{(1,8,9)}$.

$\mathrm{Na}$ vigência de embolia do líquido amniótico e/ou coagulação intravascular disseminada, a evacuação uterina deve ser imediata ${ }^{(1,8,9)}$.

O trauma fetal direto é infreqüente, já que o líquido amniótico amortece e dissipa as forças do trauma (princípio de Pascal). A lesão craniana fetal mais comum resulta da fratura da pelve materna no final da gestação, quando a cabeça do feto está insinuada, na apresentação cefálica ${ }^{(1,3,8)}$.

\subsection{Trauma abdominal penetrante}

As incidências de lesões da mãe e do filho são diametralmente opostas ${ }^{(3)}$. Em apenas $19 \%$ de todos os casos, ocorre lesão visceral materna. Por outro lado, o feto é atingido em $2 / 3$ das penetrações traumáticas do abdome. Em consequiência, ocorre uma diferença significativa de mortalidade (de 41 a $71 \%$ do feto e menos de $5 \%$ da mãe) $)^{(3)}$. Assim como na paciente não gestante, os ferimentos por arma de fogo devem ser obrigatoriamente explorados. Existem autores que adotam uma conduta seletiva nos ferimentos penetrantes por arma branca, explorando a lesão, sob anes- tesia local (se necessário, amplia-se o ferimento) e indica-se a laparotomia exploradora em todos os casos nos quais a aponeurose tenha sido violada ${ }^{(3)}$.

Em cerca de $80 \%$ das gestantes admitidas com choque hemorrágico que sobrevivem, o feto acaba indo a óbito ${ }^{(1)}$.

\section{ATENDIMENTO INICIAL}

Para um melhor resultado, tanto para a mãe, quanto para o feto, recomenda-se avaliar e ressuscitar a mãe primeiro e, então, avaliar o feto, antes que se proceda a avaliação secundária materna ${ }^{(1)}$.

\subsection{Avaliação primária}

As prioridades são as mesmas da paciente não gestante $^{(1)}$. Existem, todavia, algumas peculiaridades no atendimento à gestante:

- a paciente deve, tão logo seja descartada uma lesão da coluna cervical, ser mantida em decúbito lateral esquerdo, para que o útero não comprima a veia cava inferior, o que diminui o retorno venoso e pode agravar o choque circulatório ${ }^{(1,4,5)}$. Na suspeita de lesão cervical, a paciente deve ser mantida em posição supina, com elevação do quadril direito (6 a 10 centímetros) com o uso de coxim e o útero deve ser deslocado manualmente para a esquerda;

- devido ao maior consumo de oxigênio pela gestante, a suplementação de oxigênio deve ser prontamente instituída ${ }^{(5)}$;

- em consequiência do aumento do volume intravascular e da rápida contração da circulação uteroplacentária, desviando sangue do feto, a gestante pode perder até $35 \%$ da sua volemia antes de apresentar taquicardia, palidez, frialdade de extremidades, hipotensão e outros sinais de hipovolemia. Assim, o feto pode estar em "choque hipovolêmico", uma vez que a diminuição abrupta da volemia materna leva a um grande aumento da resistência vascular uterina, reduzindo a oxigenação fetal, independentemente de sinais vitais maternos normais $^{(1,4)}$. O uso de agentes vasopressores para a restauração da pressão arterial é contra-indicado, pois tais drogas podem diminuir ainda mais o fluxo sangüíneo fetal, já que a vasculatura placentária é extremamente sensível à estimulação por catecolaminas, levando à hipoxia fetal ${ }^{(1)}$. A gestante deve ser mantida em hipervolemia relativa e, para isso, é fundamental a monitorização precoce da pressão venosa central ${ }^{(1,5)}$. 


\subsection{Monitorização}

A mãe deve ficar em decúbito lateral esquerdo ou em posição supina com elevação do quadril direito e deslocamento manual do útero para a esquerda, dependendo da exclusão ou suspeita de lesão medular. Freqüentemente, devem ser reavaliados os sinais vitais, pressão venosa central e, de preferência, a oximetria de pulso deve ser monitorizada continuamente ${ }^{(1)}$.

No feto, os batimentos cardíacos fetais são auscultados a partir da $10^{\text {a }}$ semana de gestação com "doppler". Após 20 a 24 semanas de gestação, a monitorização deve ser contínua para se detectar sofrimento fetal agudo precocemente. A freqüência cardíaca fetal varia de 120 a 160 batimentos por minuto. Uma frequiência cardíaca fetal anormal, presença de desacelerações repetidas, ausência de aceleração ou variabilidade dos batimentos com a contração uterina pode ser um sinal de sofrimento fetal, necessitando de avaliação do obstetra ${ }^{(1)}$.

\subsection{Avaliação secundária}

Obedece a mesma seqüência adotada na avaliação da paciente traumatizada não gestante ${ }^{(1)}$.

O exame físico deve ser completo, com a avaliação da cabeça aos pés. Na gestante, também é necessária uma avaliação da altura, irritabilidade e sensibilidade uterinas, bem como a freqüência cardíaca e os movimentos fetais. O exame ginecológico deve ser completo $^{(4)}$. A presença de contrações uterinas sugere trabalho de parto prematuro. Contrações uterinas tetânicas, associadas a sangramento vaginal, traduzem descolamento prematuro de placenta normalmente implantada $^{(1,4)}$.

Um monitor uterino deve ser usado precocemente na avaliação da gestante.

Os sinais de alarme são: irritabilidade uterina, diminuição da altura uterina (inconsistente com a idade gestacional), sangramento vaginal, presença de líquido amniótico na vagina - evidenciada por um $\mathrm{pH}$ alcalino (entre 7 e 7,5) - que diagnostica ruptura da membrana amniótica; acelerações da freqüência cardíaca fetal em resposta a movimentos fetais e/ou desacelerações persistentes ou tardias, em resposta a contrações uterinas, sugerem hipoxia fetal ${ }^{(1,6)}$.

\section{EXAMES LABORATORIAIS}

Amostras sanguíneas devem ser coletadas durante o estabelecimento do acesso venoso e envia- das para: tipo sangüíneo, prova cruzada e contraprova, contagens de glóbulos brancos e plaquetas; dosagens de eletrólitos, uréia, creatinina, fibrinogênio e determinação do tempo de tromboplastina parcial ativada, tempo de protrombina, teste de Kleihauer-Betke (deteç̧ão de hemácias fetais na corrente sanguínea materna) e exames toxicológicos ${ }^{(1)}$.

\section{EXAMES RADIOLÓGICOS}

A ultrassonografia pélvica pode ser usada para detectar os batimentos cardíacos fetais e localização da placenta. Pode confirmar o diagnóstico de ruptura uterina ou descolamento da placenta ${ }^{(1,5,6)}$.

A execução dos exames radiológicos, quando estritamente necessários, não deve ser evitada. Aproximadamente $30 \%$ da radiação absorvida pela mãe é transmitida ao feto, tendo efeito cumulativo ${ }^{(2)}$. $\mathrm{O}$ risco teratogênico é maior durante a organogênese (entre a segunda e oitava semanas de vida fetal) ${ }^{(2)}$. As radiografias da coluna cervical e tórax devem ser realizadas com o útero protegido por avental de chumbo ${ }^{(5)}$.

A tomografia axial computadorizada pode ser realizada satisfatoriamente, se cortes amplos e sobrepostos forem evitados ${ }^{(5)}$. Critério e boa técnica devem ser usados para reduzir ao mínimo a dose de $\operatorname{radiação}^{(5)}$.

\section{LAVADO PERITONEAL DIAGNÓSTICO (LPD)}

A gestação não contra-indica o LPD ${ }^{(1,6,8,10,11)}$, porém a incisão deve ser realizada na linha média acima do fundo do útero. Recomenda-se o emprego da técnica $\operatorname{aberta}^{(10,11)}$.

O LPD não possibilita a avaliação de trauma dos órgãos retroperitoneais, nem das lesões intra-ute$\operatorname{rinas}^{(10,11)}$.

\section{HEMORRAGIA MATERNOFETAL}

A hemorragia maternofetal pode resultar em anemia e morte do feto. Pode, ainda, ocasionar isoimunização, se a mãe é $\mathrm{Rh}$ negativa, pois apenas $0,01 \mathrm{~mL}$ de sangue com fator Rh positivo, sensibilizará $70 \%$ das gestantes com fator $\mathrm{Rh}$ negativo ${ }^{(1,12)}$.

$\mathrm{O}$ resultado positivo do teste de KleihauerBetke indica hemorragia maternofetal. $\mathrm{O}$ teste negativo não a exclui ${ }^{(1,12)}$. 
Deve-se cogitar a terapia com imunoglobulina em todas as gestantes vítimas de trauma com fator Rh negativo, a menos que a possibilidade de lesão uterina seja remota, como no caso de traumatismo isolado de extremidades. Havendo dúvida quanto à gravidade da lesão ou à ocorrência de hemorragia maternofetal, a gestante deve receber a imunoglobulina ${ }^{(1,2,3)}$. O tratamento deve ser instituído até 72 horas após o trauma ${ }^{(1,3,12)}$.

\section{HOSPITALIZAÇÃ̃O}

A hospitalização é obrigatória nas circunstâncias que se seguem: sangramento vaginal, irritabilidade uterina, dor e sensibilidade abdominal, evidência de hipovolemia, alterações da freqüência cardíaca fetal ou ausência de batimentos cardíacos do concepto e ruptura da bolsa amniótica ${ }^{(1,3)}$.

Em pacientes com trauma abdominal e, pelo menos, 25 semanas de gestação, a monitorização fetal deve ser realizada por 24 a 48 horas $^{(5)}$.

\section{LAPAROTOMIA EXPLORADORA}

$\mathrm{Na}$ gestante traumatizada, a laparotomia não terapêutica não apresenta maior morbidade ou mortalidade do que na paciente não gestante. $O$ temor de precipitar o trabalho de parto ou causar aborto é infundado ${ }^{(6,8,9)}$.

Quando a laparotomia exploradora está indicada, o feto, geralmente, tolera bem a cirurgia e a anestesia, desde que a oxigenação materna e a perfusão uterina sejam satisfatórias ${ }^{(3,13)}$. A realização de laparotomia para tratamento de lesões traumáticas não implica necessariamente em cesárea. Muitas mães têm parto normal após a celiotomia ${ }^{(6,9,13)}$.

Quando o útero for atingido por um ferimento penetrante, provavelmente o feto estará lesado ${ }^{(3,6)}$. Se o concepto estiver vivo, a decisão de realizar a cesária deve levar em conta a idade gestacional (maturidade fetal), condições do feto avaliadas antes da cirurgia, estabilidade fisiológica da mãe e extensão da lesão uterina ${ }^{(3,13)}$.

As indicações de cesariana, no trauma, são: lesão uterina extensa, requerendo histerectomia, hemorragia uterina que não pode ser controlada, sinais de sofrimento fetal com feto potencialmente viável, necessidade de exposição cirúrgica para reparo de lesões maternas, morte materna iminente, descolamento da placenta, ruptura uterina ou embolia de lí- quido amniótico ${ }^{(5,8,9)}$. Se indicada a cesariana e houver penetração uterina, o neonatologista e o cirurgião pediátrico devem ser requisitados para avaliação especializada $^{(3)}$.

\section{PARADA CARDIORRESPIRATÓRIA NA GESTANTE}

Todas as manobras de ressuscitação cardiopulmonar convencionais devem ser realizadas sem nenhuma modificação ${ }^{(14,15,16)}$. A assistência ventilatória e a compressão torácica externa devem ser realizadas normalmente. As medidas farmacológicas convencionais também devem ser adotadas integralmente e sem qualquer restrição. Não existe contraindicação para a desfibrilação elétrica cardíaca durante a reanimação da gestante, que é empregada com os mesmos níveis de energia empregados convencionalmente ${ }^{(14 / 17)}$.

Antes da $24^{\mathrm{a}}$ semana de gestação, os objetivos da ressuscitação cardiopulmonar podem ser dirigidos exclusivamente para a sobrevivência materna, já que não existe viabilidade fetal. Após essa idade gestacional, a orientação da ressuscitação cardiopulmonar deve incluir considerações a respeito da possibilidade de sobrevivência do concepto. O ideal é que seja obtida a ressuscitação imediata, permitindo-se a manutenção do feto. Quanto menor a idade gestacional, maior é o risco perinatal do parto cesáreo de emergência, assim, a manutenção da vida materna pode ser importante para a sobrevivência fetal, independentemente da condição cerebral da gestante ${ }^{(16,17)}$.

Depois de 32 a 34 semanas de gestação, as possibilidades de ressuscitação materna e fetal são freqüentemente melhoradas pela realização do parto cesáreo de emergência, já que o esvaziamento uterino remove a compressão da aorta e da veia cava inferior, permitindo um retorno venoso adequa$\mathrm{do}^{(16,17,18)}$.

Segundo alguns autores, se, após quatro minutos de ressuscitação cardiopulmonar, não ocorrer melhora evidente e existirem evidências de deterioração fetal, é necessário um procedimento intervencionis$\mathrm{ta}^{(19)}$. Se a idade gestacional for superior a 32 semanas, a primeira prioridade é a realização do parto cesáreo. Se a idade gestacional estiver entre 24 e 32 semanas, a toracotomia e a massagem cardíaca externa devem ser consideradas e, se depois de um a dois minutos não houver êxito dessa manobra, o passo seguinte é a realização do parto cesáreo ${ }^{(19)}$. 


\section{CESÁREA PERIMORTE}

A decisão de se realizar o parto cesáreo é complexa. Devem ser levadas em consideração as circunstâncias que precipitaram a parada cardíaca materna, a idade gestacional, a possibilidade de sobrevivência fetal, o intervalo entre a parada cardíaca e o nascimento e a disponibilidade de material e pessoal treinado para o atendimento do recém-nascido $^{(17,20,21)}$.

Há poucos dados para dar suporte a cesárea perimorte na gestante com parada cardíaca por hipovolemia. O sofrimento fetal pode estar presente mesmo na gestante hemodinamicamente normal e a progressiva instabilidade materna compromete a sobrevivência fetal. No momento da parada cardíaca por hipovolemia, o feto já sofreu hipóxia prolongada $^{(1,20,21)}$.

Para outras causas de parada cardíaca, a cesárea perimorte pode ter maior sucesso ${ }^{(1,21)}$.

O nascimento que ocorre após quinze minutos da morte materna, raramente, produz recém-nascido vivo e os sobreviventes, em quase sua totalidade, apresentam graves sequielas neurológicas. Por outro lado, todas as crianças que nascem durante os primeiros cinco minutos da morte materna são vivos e saudáveis, geralmente, não apresentando qualquer seqüela neurológica. Para os recém-nascidos entre seis e quinze minutos, a taxa de sobrevivência é menor e a incidência de lesões neurológicas é de $15 \%(21,22)$.
Há relatos de casos, demonstrando que a manutenção da vida, em gestantes com morte cerebral ou em coma, permite o parto em idade gestacional maior e em situação mais favorável de sobrevivência fetal $^{(23,24)}$.

\section{VIOLÊNCIA DOMÉSTICA}

A violência doméstica é uma causa freqüente e em ascensão de lesões nas mulheres em qualquer fase de sua vida, independente do estado social, econômico e cultural ${ }^{(1,25)}$.

Alguns padrões de lesões podem sugerir a presença de violência doméstica ${ }^{(1,26,27)}$ :

- gravidade das lesões inconsistentes com a história relatada;

- depressão, diminuição da auto-estima e tentativas de suicídio;

- procura freqüente por atendimento médico de emergência;

- sintomas sugestivos de abusos de drogas;

- maridos ou companheiros que insistem em estar presentes na anamnese e no exame físico, além de tentar monopolizar a discussão;

- insistência em assumir a culpa pelas lesões sofridas.

Os casos suspeitos de tal violência devem ser encaminhados ao serviço social e à Delegacia de Defesa da Mulher.

PEREIRA Jr. GA; HAIKEL Jr. LF; ATIQUE JMC; NAKAMURA EJ; BASILE-FILHO A \& ANDRADE JI. Trauma in pregnancy. Medicina, Ribeirão Preto, 32: 282-289, july/sept. 1999.

ABSTRACT: The priorities for management of the pregnant patient are no different than those of the nonpregnancy patient. It is important to follow a methodic evaluation that ensures complete assessment and stabilization of the mother. The mother should be stabilized before the stabilization of the fetus. The physiologic and anatomic changes during the pregnancy can influence outcome. Continuous fetal and maternal monitoring may be necessary. This article discuss about blunt and penetrating abdominal trauma, peritoneal lavage and indications of exploratory laparotomy. Cardiopulmonary resuscitation in pregnancy and perimortem cesarean delivery is considered. Indicators may suggest the presence of domestic violence and should serve to initiate further investigation.

UNITERMS: Injuries. Pregnancy. Cesarean Section. Fetal Death. Abdominal Injuries. Domestic Violence. 


\section{REFERÊNCIAS BIBLIOGRÁFICAS}

1 - ADVANCED TRAUMA LIFE SUPPORT. Instructor Manual. Committee on Trauma. American College of Surgeons, Chicago, p. 377-387, 1997.

2 - SHAPIRO MJ; DURHAM RM \& MAZUSKI JE. Abdominal and pelvic trauma. In: AYRES SM; GRENVIK A; HOLBROOK PR \& SHOEMAKER WC. Textbook of critical care, $3 \mathrm{r}$ ed. WB Saunders, ed. Philadelphia, p. 1439-1448, 1995.

3 - HANKINS GDV; BARTH JR WH \& SATIN AJ. Critical care medicine and the obstetric patient. In: AYRES SM; GRENVIK A; HOLBROOK PR \& SHOEMAKER WC. Textbook of critical care, 3rd ed. WB Saunders, Philadelphia, p. 50-63, 1995.

4 - ADVANCED TRAUMA LIFE SUPPORT. Instructor Manual. Committee on Trauma. American College of Surgeons, Chicago, p. 1-406, 1993.

5 - KNUDSON P. Trauma and pregnancy. Trauma manual of San Francisco General Hospital. University of California, San Francisco, 1995.

6 - ESPOSITO TJ; GENS DR; SMITH LG \& SCORPIO R. Evaluation of blunt abdominal trauma occorring during pregnancy. J Trauma 29: 1628-1632, 1989.

7 - SCHOENFELD A; ZIV E; STEIN L; ZAIDEL D \& OVADIA J. Seat belts in pregnancy and the obstetrician. Obstet Gynecol Surg 42: 275-282, 1987.

8 - PERLMAN MD; TINTINALLI JE \& LORENZRP. Blunt trauma during pregnancy. N Engl J Med 323: 1606-1613, 1991.

9 - SORENSEN VJ; BIVINS BA; OBEID FN \& HORST HM. Management of surgical emergencies in pregnancy. Am Surg 56: 245-250, 1990.

10 - ANDRADE JI; STRACIERI LDS \& MORAIS EN. Lavado peritoneal ou punção simples? In: PAULA CASTRO L \& SAVASSI ROCHA PR. Tópicos em gastroenterologia 3. Medsi, Rio de Janeiro, p. 419-430, 1992.

11 - ANDRADE JI \& STRACIERI LDS. Lavado peritoneal diagnóstico. In: SAVASSI ROCHA PR; ANDRADE JI \& SOUZA C. Abdome agudo: diagnóstico e tratamento. 2a. ed. Medsi, Rio de Janeiro, p. 25-29, 1993.

12 - ROSE PG; STROHN PL \& ZUSPAN FP. Fetomaternal hemorrhage following trauma. Am J Obstet Gynecol 153: 844847, 1985.

13 - KISSINGER DP; ROZYCKI GS; MORRIS JA Jr; KNUDSON MM; COPES WS; BASS SM; YATES HK \& CHAMPION HR. Trauma in pregnancy - predicting pregnancy outcome. Arch Surg 126: 1079-1086, 1991.
14 - SONGSTER VL \& CLARK SL. Cardiac arrest in pregnancy. What to do ? Contemp Obstest Gynecol 27: 141-155, 1985.

15 - LEE RV; ROGERS BD; WHITE LM \& HARVEY RC Cardiopulmonar resuscitation of pregnant women. Am $\mathbf{J}$ Med 81: 311-318, 1986.

16 - ADVANCED CARDIAC LIFE SUPPORT. Special Resuscitation Situations, American Heart Association, Chicago, 10: $1-27,1994$

17 - LICHTENSTEIN A \& BORN D. Situações especiais da reanimação cardiorrespiratória. Rev Soc Cardiol São Paulo 8: 779-797, 1998.

18 - GOODWIN P \& PEARE J. The human wedge: a maneuvre to relieve aortocaval compression during resuscitation in late pregnancy. Anaesthesia 47: 433-444, 1992.

19 - LEE RV \& MEZZARDI FC. Cardiopulmonary resuscitation of pregnant women. In: ELKAYAM U \& GLEICHER N, eds. Cardiac problem in pregnancy. Alan R. Liss, New York; p.307-319, 1990.

20 - KATZ VL; DOTTERS DJ \& DROEGEMUELLER W. Perimortem cesarean delivery. Obstet Gynecol 68: 571-576, 1986

21 - STRONG JR TH \& LOWE RA. Perimortem cesarean section. Am J Emerg Med 7: 487-497, 1989.

22 - LOPEZ ZENO J; CARLO WA; O'GRADY JP \& FANAROFF AA. Infant survival following delayed postmortem cesarean delivery. Obstet Gynecol 76: 991-992, 1990.

23 - DILLON WP; LEE RV; TRONOLONE MJ; BUCKWALD S \& FOOTE RJ. Life support and maternal brain dath during pregnancy. JAMA 248: 1089-1091, 1982.

24 - HILL LM; PARKER D \& O'NEILL BP. Management of maternal vegetative state during pregnancy. Mayo Clin Proc 69:469-472, 1985.

25 - ROBERTS GL; O'TOOLE BI; RAPHAEL B; LAWRENCE JM \& ASHBY R. Prevalence study of domestic violence victims in an emergency department. Ann Emerg Med 27: 741753, 1996.

26 - OLSON L; ANCTIL C; FULLERTON L; BRILLMAN J; ARBUCKLE J \& SKLAR D. Increasing emergency physician recognition of domestic violence. Ann Emerg Med 27: 741-746, 1996.

27 - DUNNEGAN SW. Violence, trauma and substance abuse. J Psychoactive Drugs 29: 345-351, 1997.

Recebido para publicação em 11/08/99

Aprovado para publicação em 14/09/99 